\title{
Surface Modifications of an Organic Polymer-Based Microwire Platform for Sustained Release of an Anti-Inflammatory Drug
}

Chuan Liu,${ }^{a} \neq$ Michelle A. Nguyen, ${ }^{b, \neq}$ Anabel Alvarez-Ciara,${ }^{b}$ Melissa Franklin,${ }^{b}$ Cassie Bennett ${ }^{b}$ Justin B. Domena,${ }^{a}$ Noah C. Kleinhenz, ${ }^{a}$ Gabriel A. Blanco Colmenares, ${ }^{a}$ Sebastian Duque, ${ }^{a}$ Aisha F. Chebbi, ${ }^{a}$ Brianna Bernard, ${ }^{a}$ Jean-Hubert Olivier,${ }^{a,{ }^{*}}$ Abhishek Prasad ${ }^{b, c^{*}}$

a Department of Chemistry, University of Miami, Cox Science Center, 1301 Memorial Drive, Coral Gables, FL 33146, USA

${ }^{\mathrm{b}}$ Department of Biomedical Engineering, University of Miami, 1251 Memorial Drive, Coral Gables, FL 33146, USA

${ }^{\mathrm{c}}$ The Miami Project to Cure Paralysis, University of Miami, Miami, FL 33136, USA

₹These authors contributed equally.

\section{Table of Contents}

1. Synthesis of PEG 3400 Hydrogel (p. S2)

2. Mechanical Properties of $\mathrm{PEG}_{3400}$ Hydrogel (p. S3)

3. Microscopic Characterization of the Functionalized Wires (p. S4-S10)

4. Additional Drug Release Profiles (p. S11-S12)

5. DLS Profile of DEX Aggregates (p. S13)

6. Movies Information (p. S14)

7. References (p. S15) 


\section{Synthesis of PEG $_{3400}$ Hydrogel.}

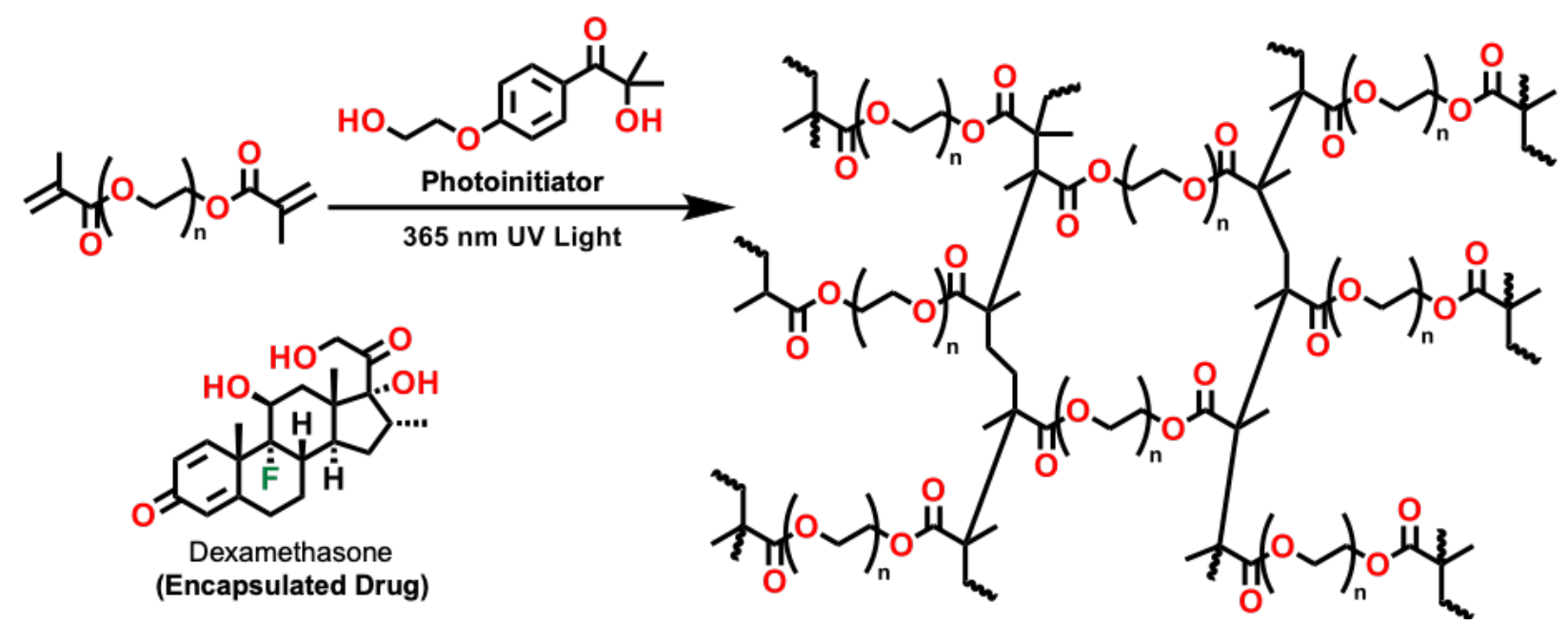

Scheme S1. Reaction scheme for photopolymerization of PEGDMA precursors. Chemical structures of the hydrogel precursor, the photoinitiator, and the drug studied are shown. The 3D polymer network formed is schematized here in a $2 \mathrm{D}$ manner. 


\section{Mechanical Properties of PEG $_{3400}$ Hydrogel.}

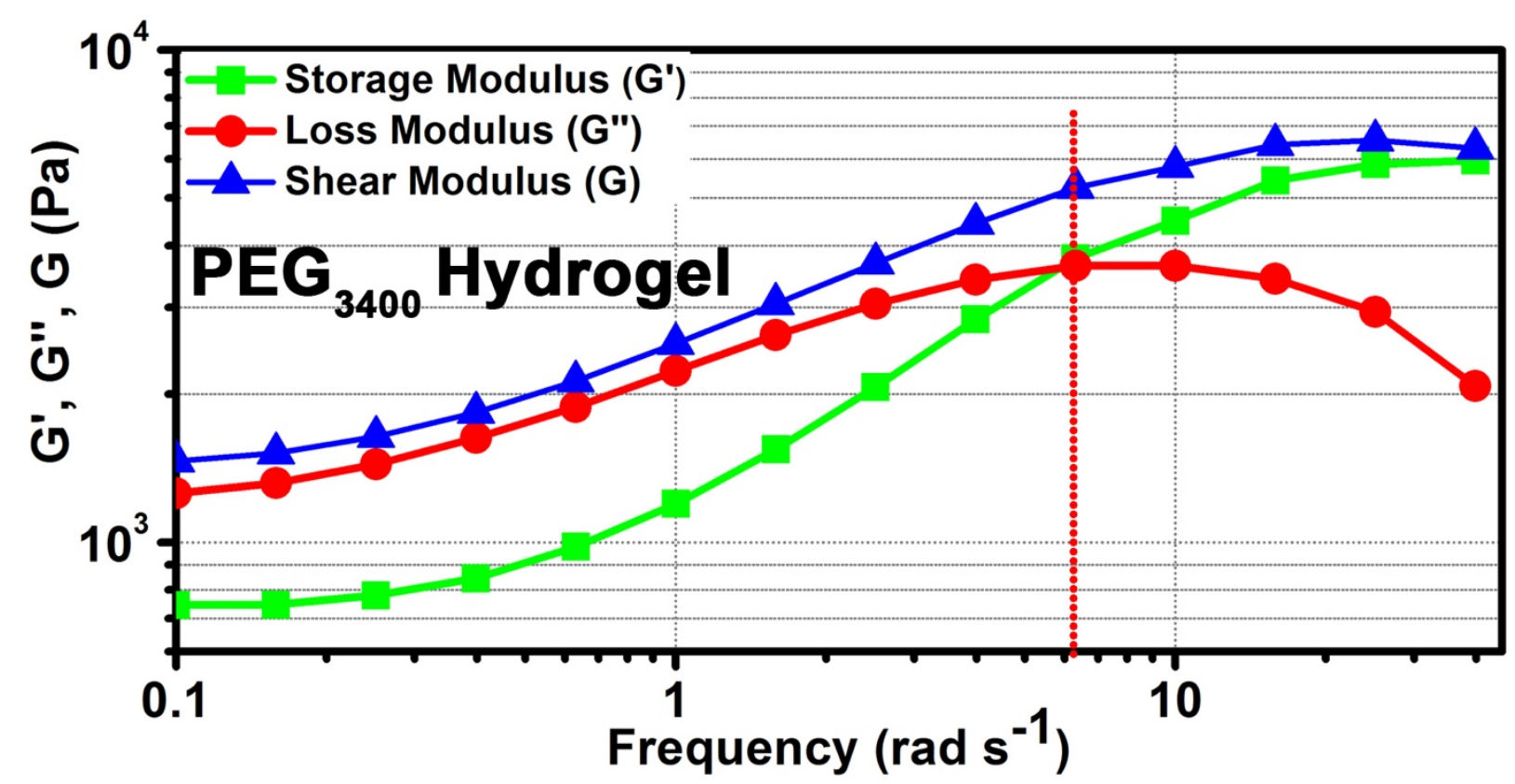

Figure S1. Rheology frequency sweep results for Dex8.9-PEG 3400 hydrogel block.

The Young modulus is calculated using the following equation:

$$
\mathrm{E}=2 \mathrm{G}(1+\mathrm{v})(\text { eq. } \mathrm{S} 1)
$$

Where E is the Young's modulus, G the shear modulus, and v the Poisson's ratio of the materials. Data were averaged based on three replicates. Dex8.9-PEG ${ }_{3400}$ hydrogel block is characterized by a Young's modulus of $25.7 \mathrm{kPa}$. 
3. Microscopic Characterization of the Functionalized Wires.

\section{Pt-Ir-COOH}

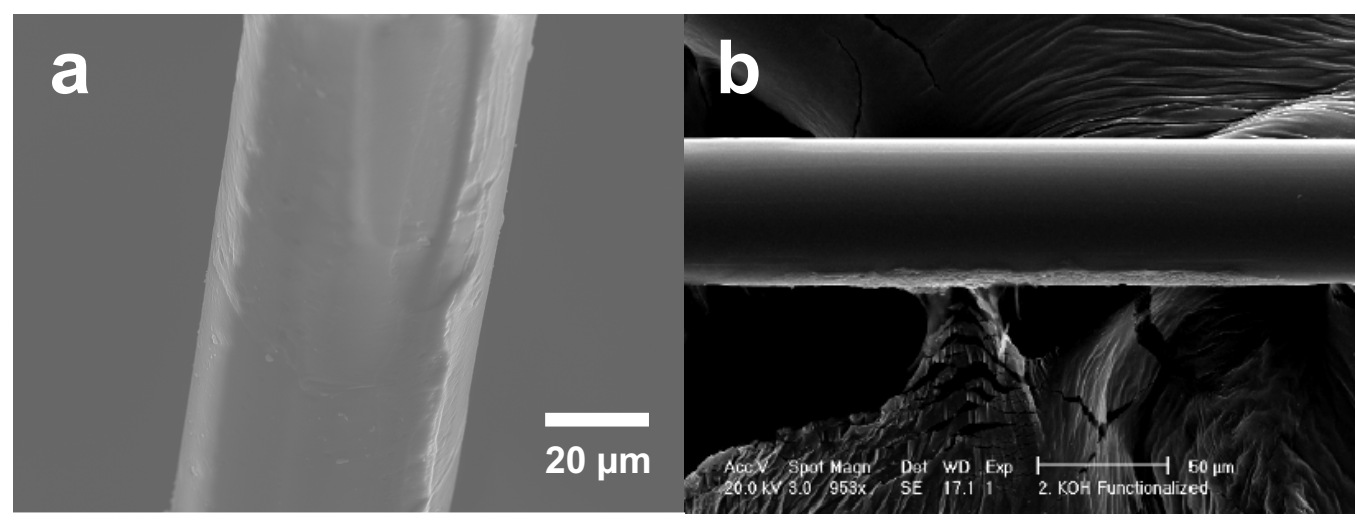

\section{Pt-Ir-OTEG}

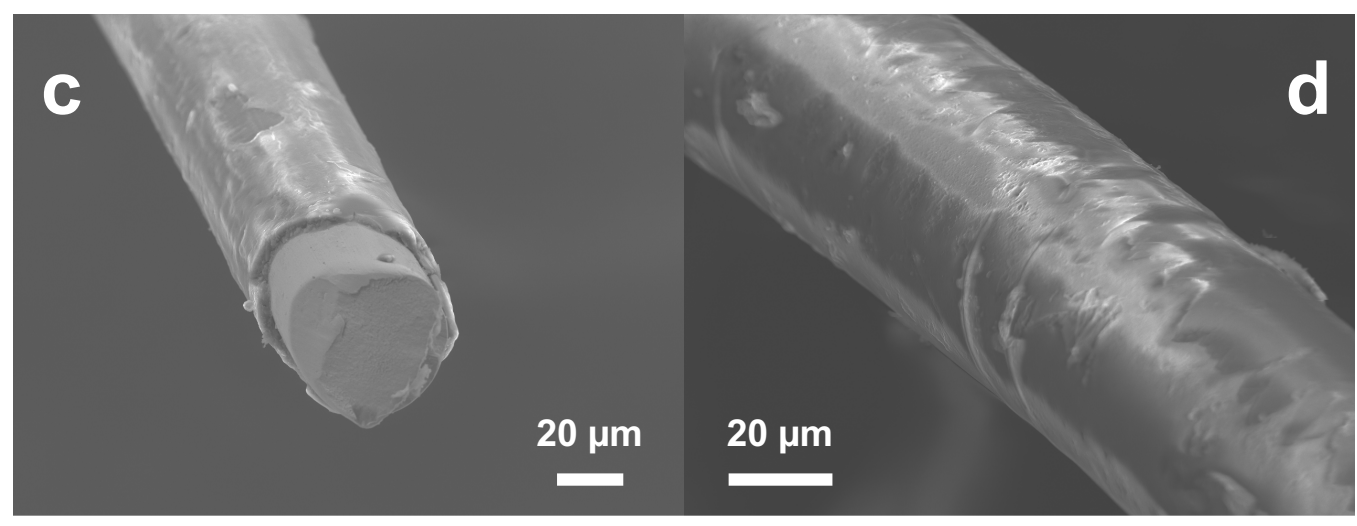

\section{Pt-Ir-MAC}

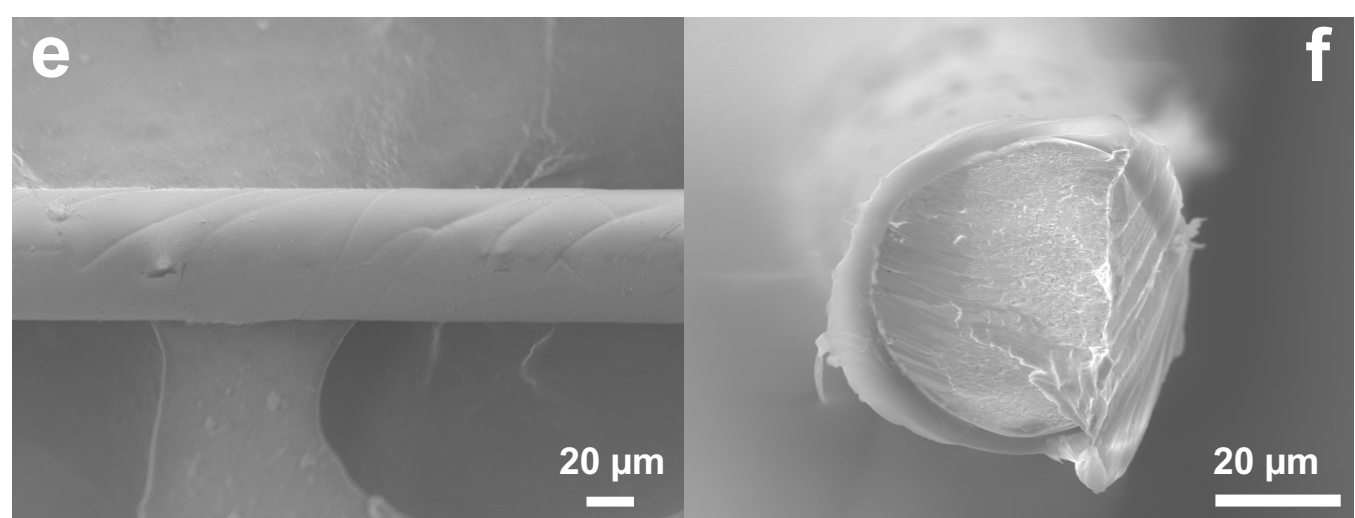

Figure S2. Additional SEM images of: (a-b) carboxylic acid functionalized microwire (Pt-IrCOOH), (c-d) triethylene glycol functionalized microwire (Pt-Ir-TOHEG), and (e-f) methacrylate-functionalized microwire (Pt-Ir-MAC). 

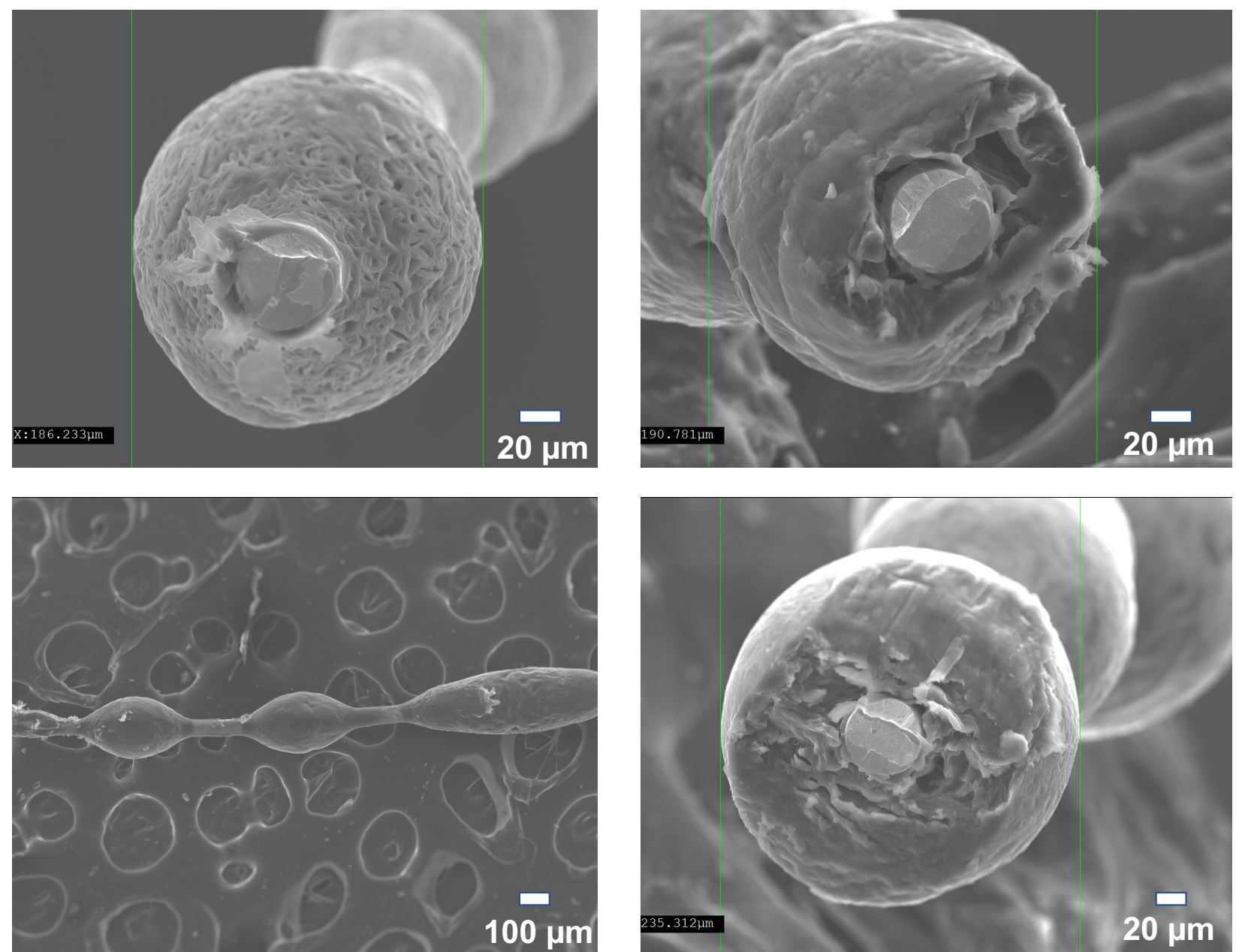

Figure S3. SEM images of dry hydrogel-functionalized microwires fabricated using the painting method and the following hydrogel precursor solution: $8.9 \%$ Dex w/w, 50\% w/w PEGDMA 3400 , EtOH:PBS 1:1. 

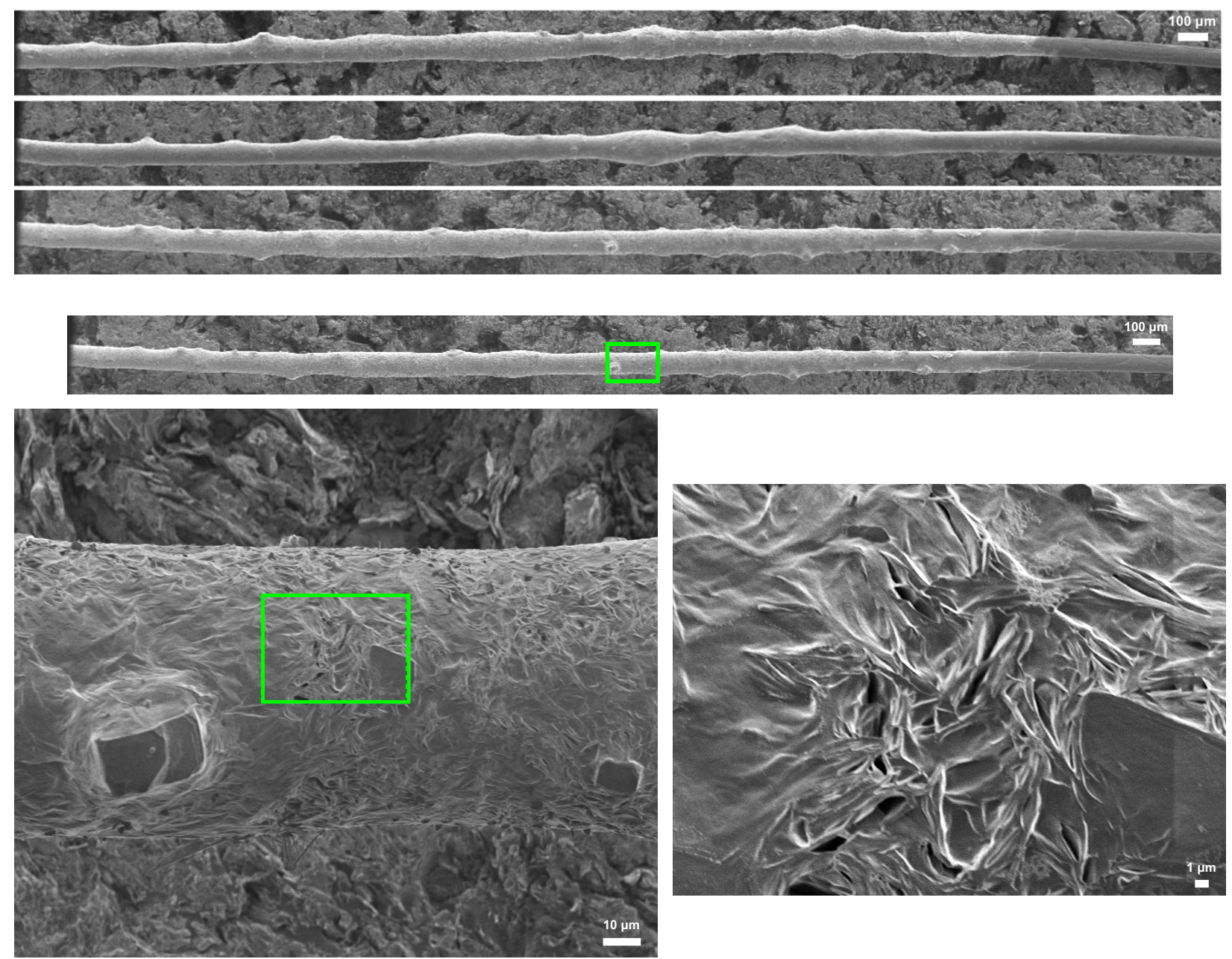

Figure S4. Additional SEM images of functionalized drug-loaded hydrogel microwires Dex-Pt-

Ir-PEG 3400 fabricated with 50\% w/w PEGDMA, 8.9\% w/w Dex, and EtOH/PBS (3:1). 

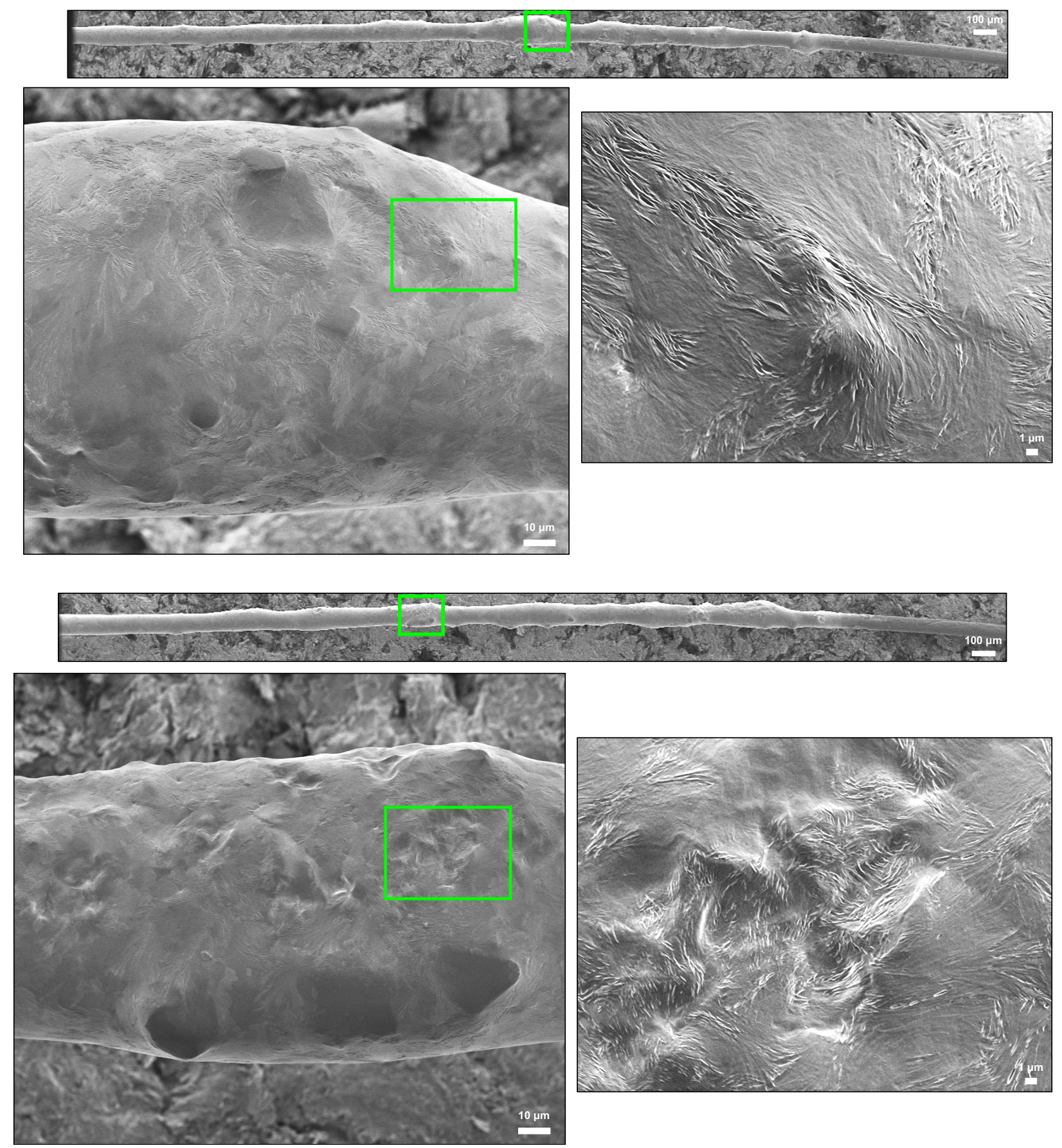

Figure S5. Additional SEM images of functionalized drug-loaded hydrogel microwires Dex-Pt-

Ir-PEG 3400 fabricated with 50\% w/w PEGDMA $3400,8.9 \%$ w/w Dex, and EtOH/ $\mathrm{H}_{2} \mathrm{O}(3: 1)$. 


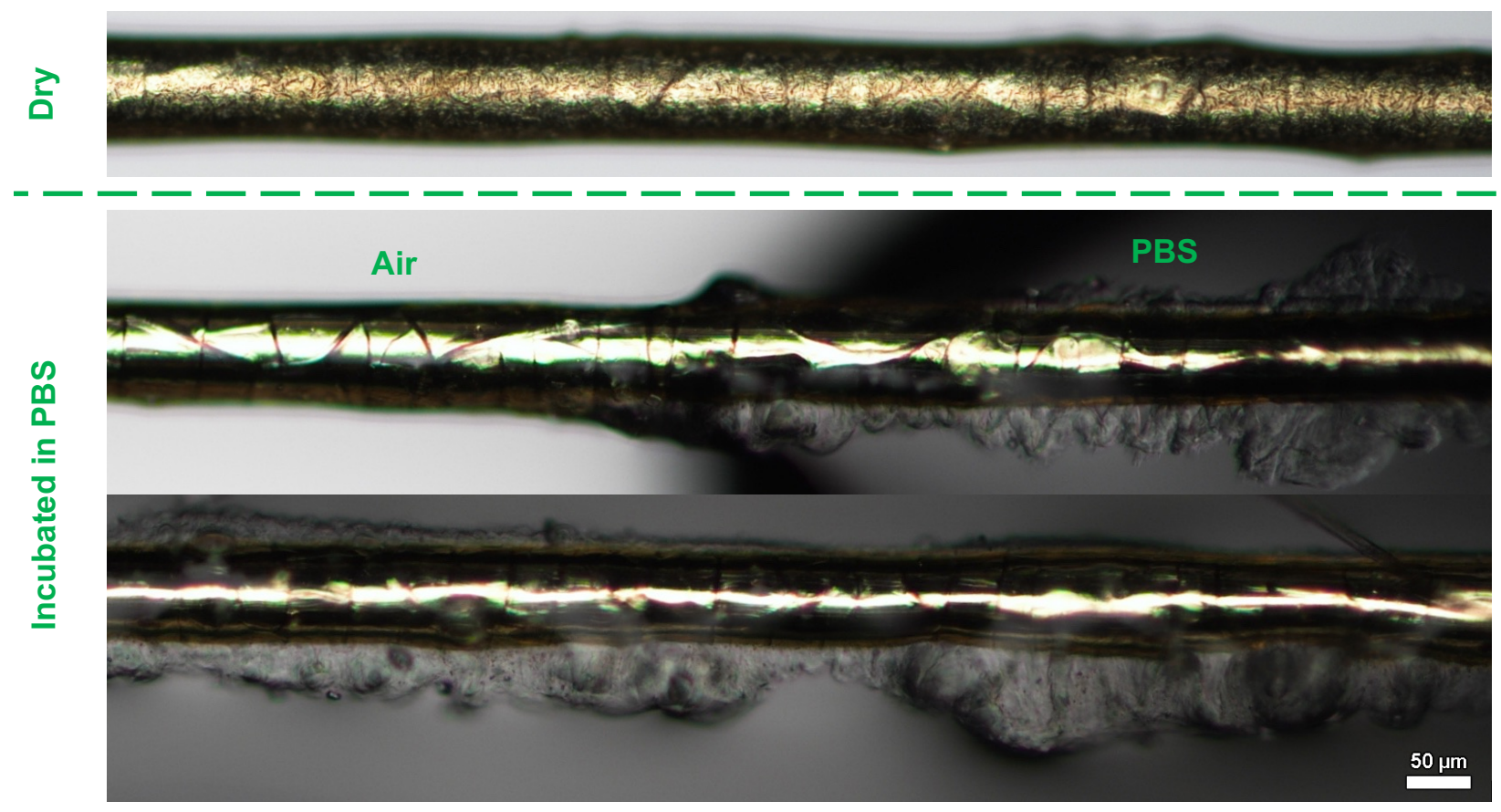

Figure S6. Additional optical microscopy images of functionalized drug-loaded hydrogel microwires Dex-Pt-Ir-PEG 3400 fabricated with $50 \%$ w/w PEGDMA $3400,8.9 \%$ w/w Dex, and $\mathrm{EtOH} / \mathrm{H}_{2} \mathrm{O}(3: 1)$. 

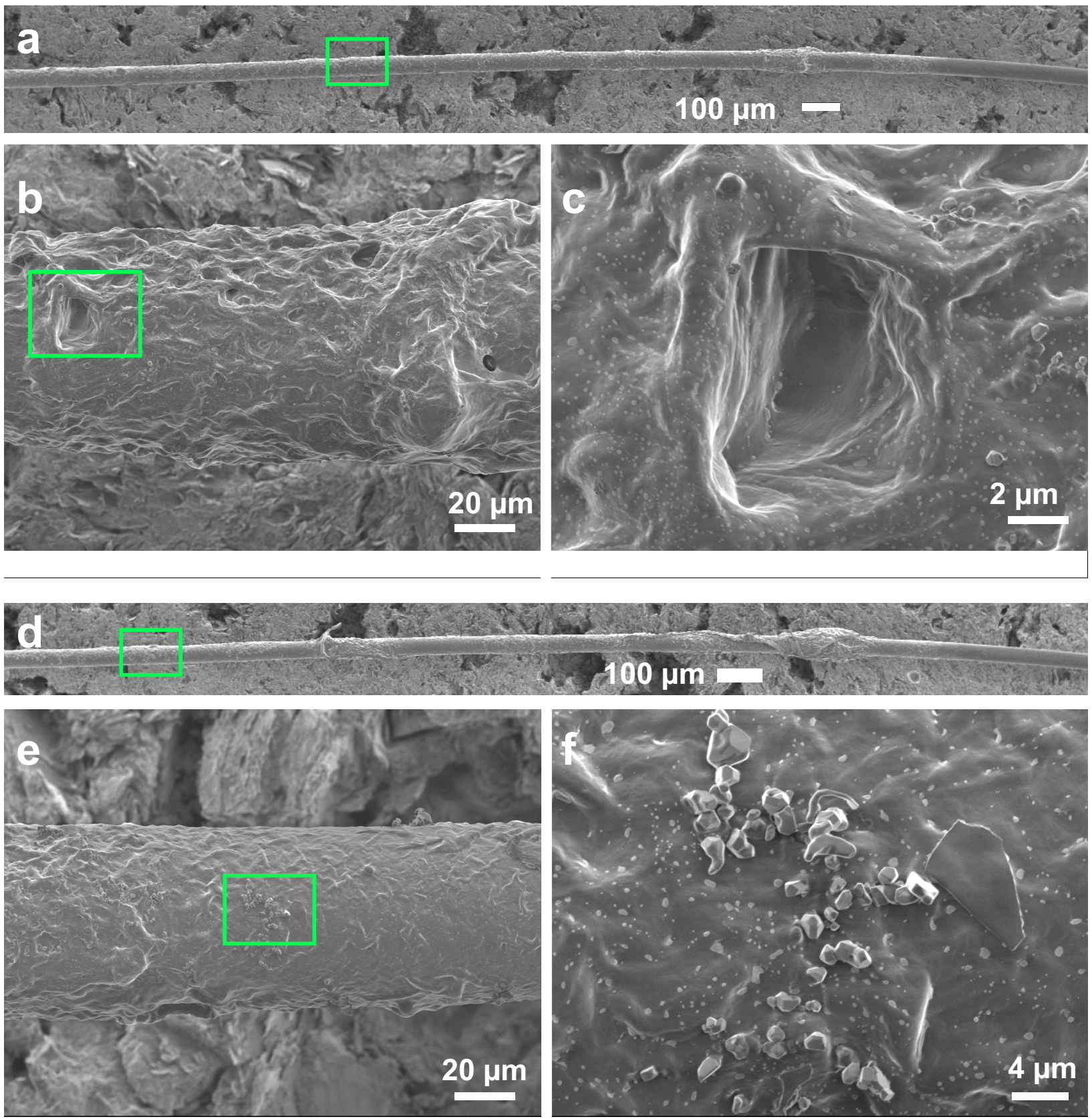

Figure S7. Additional SEM images of functionalized drug-loaded hydrogel microwires Dex-Pt-

Ir-PEG 3400 after immersion in PBS buffer. 

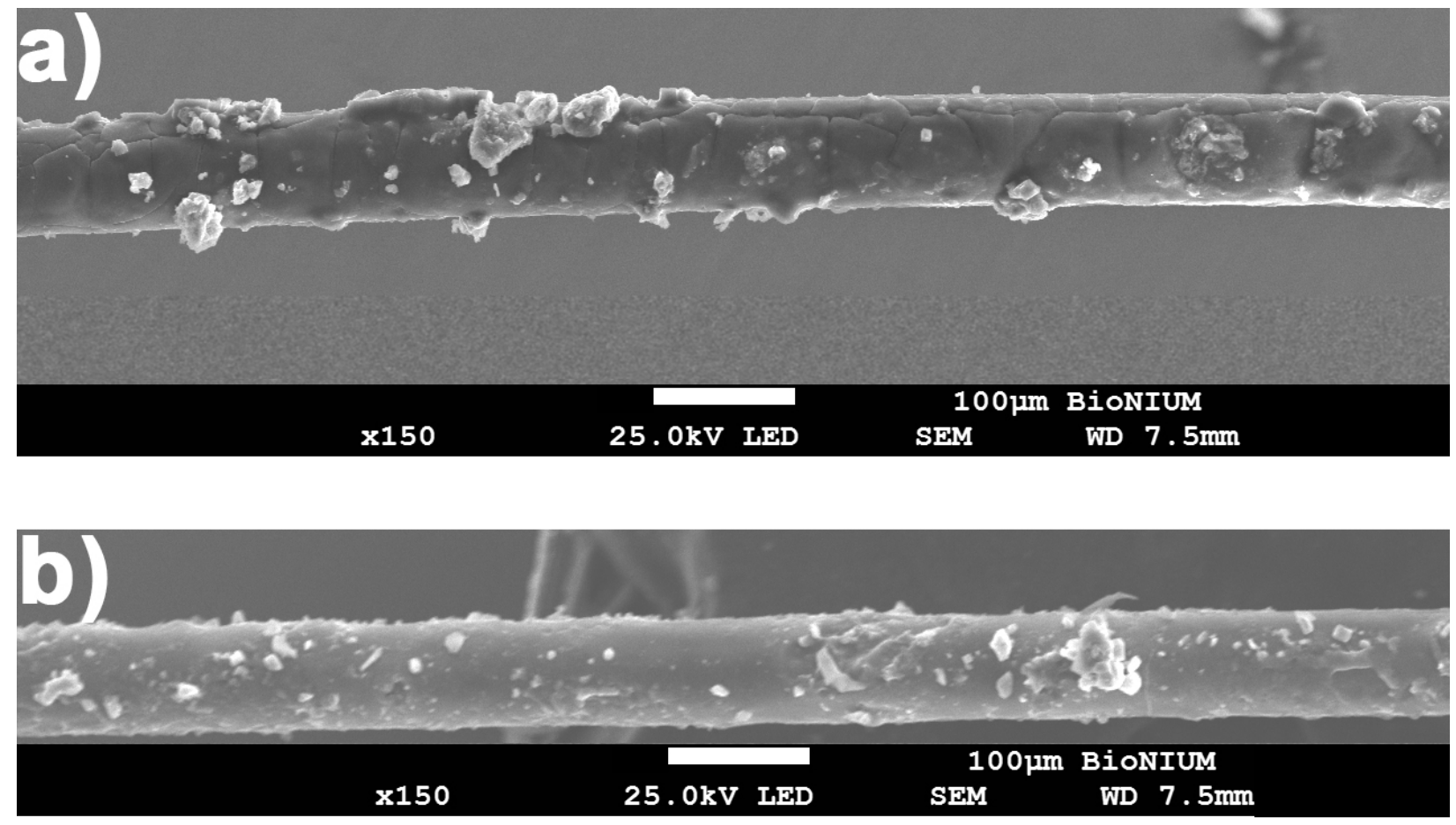

Figure S8. Additional SEM images of functionalized drug-loaded hydrogel microwires Dex-Pt-

Ir-PEG 550 fabricated with 50\% w/w PEGDMA $3400,8.9 \%$ w/w Dex, and EtOH/ $\mathrm{H}_{2} \mathrm{O}(3: 1)$. 


\section{Additional Drug Release Profiles}
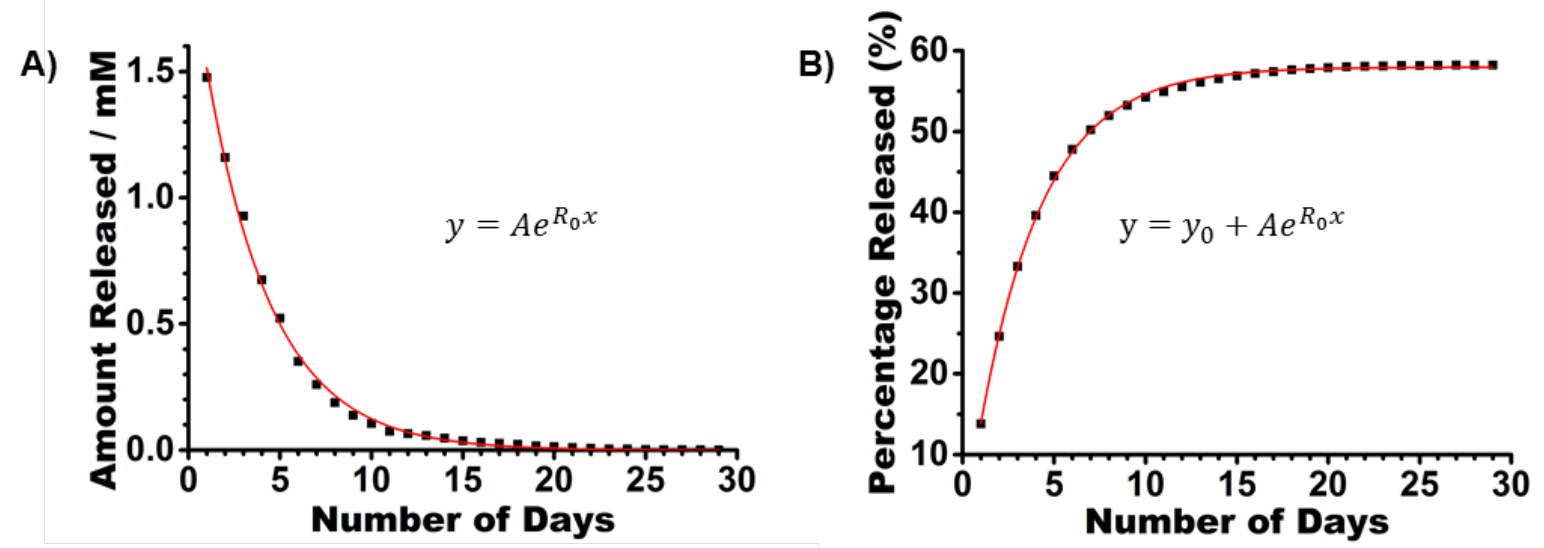

Figure S9. A) Dexamethasone release profile of Dex1.1-PEG 3400 hydrogel. Data points were fitted exponentially, where $A=2.00 \mathrm{mM}$ and $R_{0}=-0.277 \mathrm{~d}^{-1}$. B) Percentage of the total amount of loaded drug released over time for the Dex1.1-PEG 3400 hydrogel. Data points were fitted exponentially, where $y_{0}=58.0, A=-58.7$ and $R_{0}=-0.287 \mathrm{~d}^{-1}$. Note: the hydrogel fabrication method and the drug release test method are the same as described in the main text except that this gel was air-dried instead of being dried under vacuum thoroughly. 


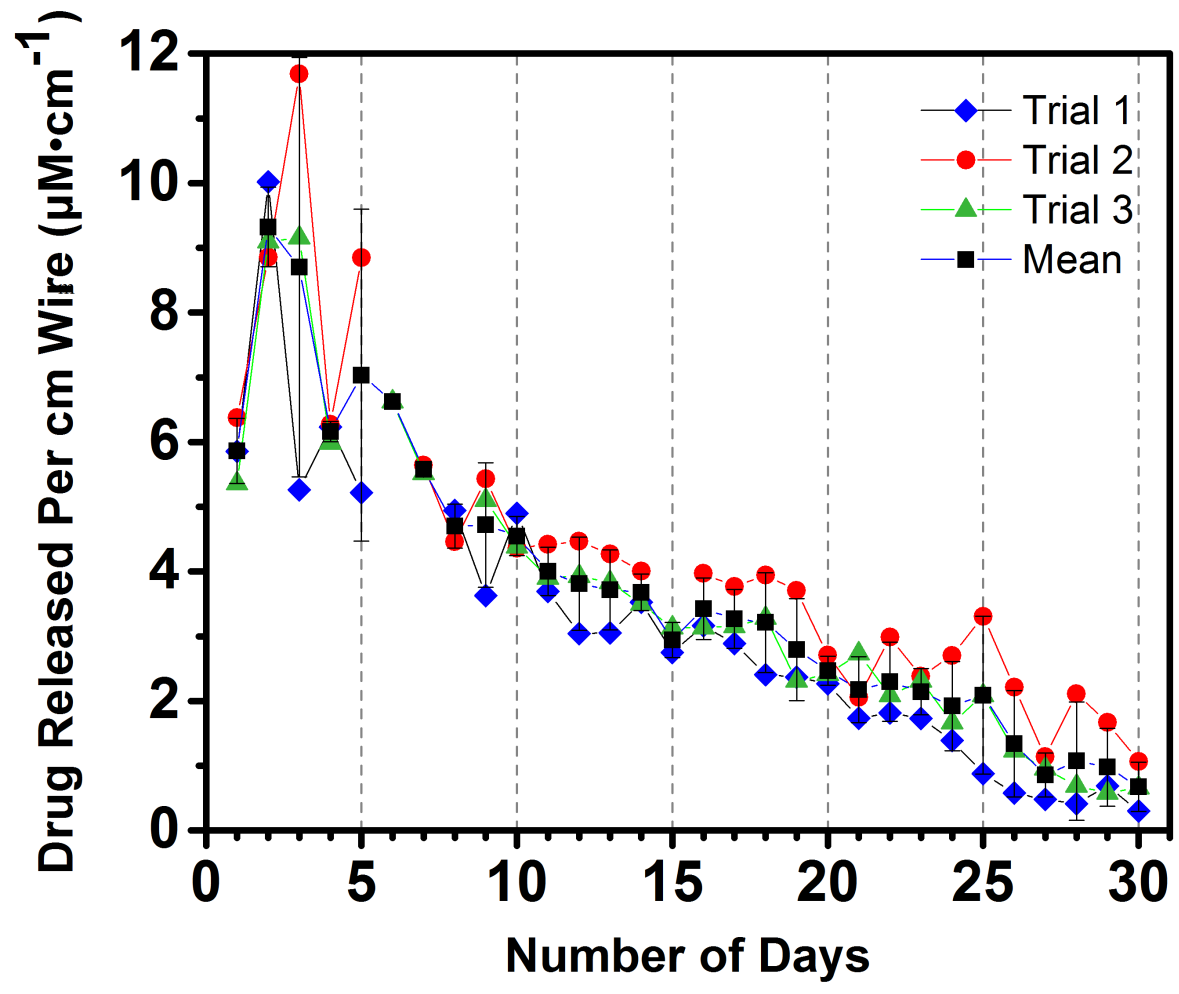

Figure S10. DEX release profile of Pt-Ir microwires coated with drug-loaded PEGDMA (MW $\approx$ 550) hydrogel, where the y-axis is the molarity of Dex released in the supernatant per centimeter of wire. All three trials were done in parallel, and the mean and standard deviation values were calculated and are presented here as well. 
5. DLS profile of DEX aggregates.

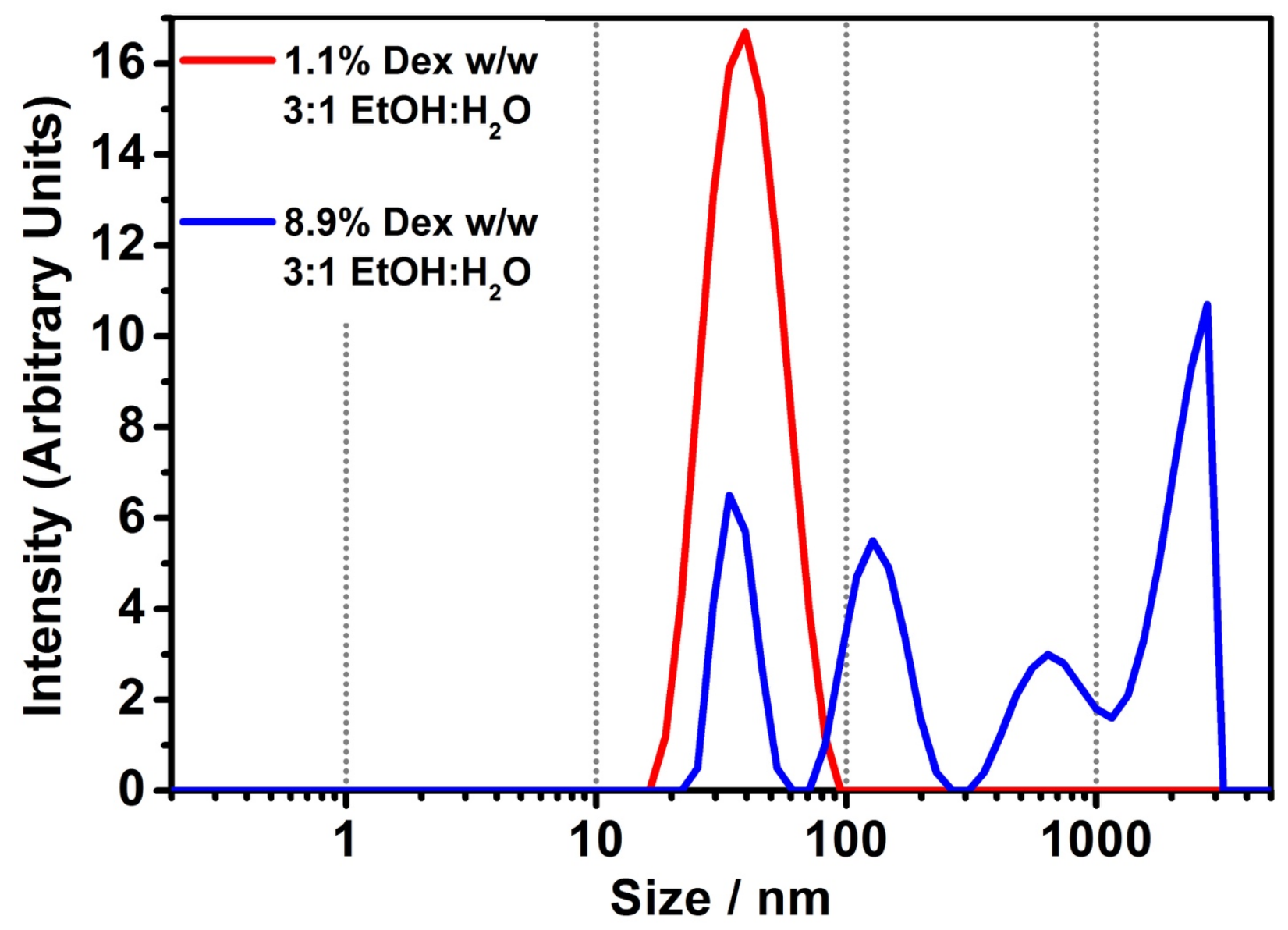

Figure S11. Average particle sizes of Dex aggregates as a function of Dex loading in 3:1 EtOH: $\mathrm{H}_{2} \mathrm{O}$ recorded using dynamic light scattering experiments. 


\section{Movies Information}

Movie S1. Optical microscopy recording of a functionalized drug-loaded hydrogel microwire fabricated with 50\% w/w PEGDMA, 8.9\% w/w Dex, and EtOH/PBS (3:1) being immersed in 1x PBS.

Movie S2. Optical microscopy recording of a functionalized drug-loaded hydrogel microwire fabricated with 50\% w/w PEGDMA, 8.9\% w/w Dex, and EtOH/ $\mathrm{H}_{2} \mathrm{O}(3: 1)$ being immersed in 1x PBS. 


\section{References}

1. Bennett, C.; Samikkannu, M.; Mohammed, F.; Dietrich, W. D.; Rajguru, S. M.; Prasad, A., Blood brain barrier (BBB)-disruption in intracortical silicon microelectrode implants. Biomaterials 2018, 164, 1-10.

2. Bennett, C.; Mohammed, F.; Alvarez-Ciara, A.; Nguyen, M. A.; Dietrich, W. D.; Rajguru, S. M.; Streit, W. J.; Prasad, A., Neuroinflammation, oxidative stress, and blood-brain barrier (BBB) disruption in acute Utah electrode array implants and the effect of deferoxamine as an iron chelator on acute foreign body response. Biomaterials 2019, 188, 144-159.

3. Livak, K. J.; Schmittgen, T. D., Analysis of relative gene expression data using real-time quantitative PCR and the 2(T)(-Delta Delta C) method. Methods 2001, 25 (4), 402-408. 